\title{
A
}

Acta HealthMedica

Volume: 2, Issue: 1, January-March 2017, Pages: 151, DOI: http://dx.doi.org/10.19082/ah151

\section{MHEALTH TECHNOLOGY FOR DEAF AND HEARING LOSS INDIVIDUALS}

\author{
Farzaneh Kermani ${ }^{1}$, Azam Orooji ${ }^{2}$, Alireza Borhani ${ }^{3}$
}

1: PhD Student of Medical Informatics, School of Health Management and Information Sciences, Iran University of Medical Sciences, Tehran, Iran

2: PhD Student of Medical Informatics, School of Health Management and Information Sciences, Iran University of Medical Sciences, Tehran, Iran

3: MSc of Medical Informatics, School of allied medical science. Tehran University of Medical Science, Tehran, Iran

Correspondence:

Azam Orooji, Tel: +98-2188794301, E-mail: orooji.a@tak.iums.ac.ir

\section{TYPE OF ARTICLE: CONFERENCE ABSTRACT}

\begin{abstract}
Introduction: Hearing loss is one of the most commonly occurring sensory deficits affecting communities and is ranked as the third most prevalent nonfatal disabling condition. Using mobile devices in medical practice has become an emerging trend. mHealth, or mobile health, is defined by the WHO as "medical and public health practice supported by mobile devices, such as mobile phones, patient monitoring devices, personal digital assistants (PDAs) and other wireless devices." According to the latest update (2004) of the WHO Global Burden of Disease (GBD), hearing loss is one of the eight prevalent health conditions for which mobile applications are dedicated. The aim of this paper is to investigate special aspects of using mHealth and requirements for providing mobile-based services for helping hearing-loss individuals.

Methods: The articles published between 2004 and 2016 in the PubMed database were reviewed by using "mobile health," "mHealth," "mobile phone," "smart phone," and "cellular phone" in combination with "deaf" and "hearing loss" keywords. Forty-three English articles were found in which, after removing duplicates and nonavailable full-text papers, title and abstract articles were screened by two reviewers; at the end, 18 publications were included in this article based on eligibility criteria.

Results: Mobile tools are used for the hearing impaired, language dysfunction, and foreigners in emergency situations. Deaf people typically use text communication such as SMS, MMS, fax, email, fire emergency and doorbell announcements, or video phones on their mobile phone, whereas over $90 \%$ of them exchange content through a SMS. However, different mobile or tablet audiometer apps can be used for ear and hear screening by physician or play as self-assessment hearing tools for patients. Calculation of a hearing handicap sensitivity test with mobile devices and development of mobile communication tools to support deaf persons in understanding directions for medication are some utilized methods of mHealth in helping deaf individuals. Also, it is important to consider some essential needs and requirements in developing these applications. Users have needs of items such as social, communication, entertainment, and safety demands. Moreover, general requirements such as touch screens, handwriting recognition, cameras and displays, visual and tactile presentations, text-to-speech and speech-to-text converters, with standard and consistent designs are among the most important issues to address.

Conclusion: Using mHealth apps is inexpensive and presents opportunities for physicians to integrate technology in clinical practice. Due to the prominent burden of individuals with hearing loss and limitations in providing services for them, new methods are needed to support them in developing countries.
\end{abstract}

KEYWORDS: Mobile health, MHealth, Mobile Phone, Deaf, Hearing loss

\section{Abstracts of First National Congress of Medical Informatics, Mashhad, Iran, February 2017}

(C) 2017 The Authors. This is an open access article under the terms of the Creative Commons Attribution-NonCommercialNoDerivs License, which permits use and distribution in any medium, provided the original work is properly cited, the use is non-commercial and no modifications or adaptations are made. 